\title{
Migrant worker and Mental health in Nepal
}

Nepal is one of the largest exporters of cheap and low skilled labor to thriving economies of the world especially the gulf countries. Prevalent poverty, political and financial instability, limited job opportunities, government's inability to boost up the agricultural sector and enticement to earn some bulk cash in quick time are some of the defining reasons compelling common man to seek drastic measures. The government statistics suggest that every year over 300,000 Nepalese go abroad for employment. But the cause of concern is that the majority of the migrants is semi skilled or low skilled, who have at maximum attended secondary to higher secondary level education. The scenario makes them to accept jobs that have risk prone working conditions, which are rejected by the local workers. As a result we frequently hear tragic news about accidents, disabilities, physical and mental abuse leading to mental trauma. The news of suicides and suicidal attempts by the female workers working as housemaids as result of physical and sexual abuse is even more heart breaking. The government of Nepal seems to focus only on the ensuring boosting their economy with the remittance which makes up $22 \%$ of Gross Domestic Product (GDP), closing eyes on the workers health and well being.

Migrating to different country in it self leads to significant changes in personal, family and societal life of the migrant worker. Moving to a totally different country leading to cultural shock, loneliness, home sick and stress at the work place can all lead to mental health problems. The mental health may not be only limited to the migrant but the long separation also takes its toll on the immediate members of the family. The parents of the migrant work may lack able hands to help them out with the routine activities and personal care leading to depression. The wife/husband may find it hard to undertake different household activities on his $\backslash$ her own. The children may miss their father/mother or both which will ultimately effect the emotional attachment and psychic of their offspring. Psychiatric problems such as somatoform disorder, dissociative disorder, psychosis, organic psychosis, bipolar affective are found prominent not only in the migrants but also in their family members.

The government does have a system of certifying the migrant workers as mentally sound. But the preimmigration health check up often has one line statement where it states that the individual is mentally sound which is almost always conducted by physicians who do not have any experience in mental health. In clinical practice it is not unusual to find clients who have returned from abroad with significant mental health problems. Hence with ever increasing outflow of workers time has come to put in place a system of pre-screening and treatment if needed on any mental health problem through a certified psychiatrist. As the workers go through significant change/stress when moving to work abroad, there should also be sufficient mental health services available to them at the country of destination. Similarly, when a migrant worker returns back to Nepal, he/she may have problems reintegrating back to the society as a result of long stay in foreign land. Therefore it would be advisable that Government of Nepal work towards establishing a system whereby they could have access to sufficient mental health services to minimize the impact on them, family and the society at large. The working population is the most valuable asset in achieving our national goal. A return of a worker due to mental health problem causes severe financial stress to an individual and to a country as a whole. Therefore it would be at our best interests to move ahead with ensuring mental well being of our migrant workers, which not only is financially rewarding but also socially beneficial. 